\title{
Kinetics of desorption from a heterogeneous surface
}

\author{
A. Córdoba and J. J. Luque \\ Departamento de Termología, Facultad de Física, Universidad de Sevilla, \\ Sevilla, Spain \\ (Received 9 April 1982; revised manuscript received 26 July 1982)
}

\begin{abstract}
Mathematical equations governing kinetics of desorption from heterogeneous surfaces are derived from a master equation with the assumption that only nearest-neighbor adatoms interact. A number of cases are analyzed: random, periodical, and patchwise distributions of sites characterized by different activation energies. In general, the kinetic equations obtained must be solved numerically. We have performed numerical calculations for several specific cases and analyzed the influence exerted by heterogeneity and lateral interaction between adatoms on the desorption rate $d \Theta / d T$. The results obtained make it clear that, due to lateral interaction, the desorption curves are very sensitive to the way in which heterogeneities are distributed on the surface (periodically, patches with different sizes, etc.).
\end{abstract}

\section{INTRODUCTION}

In general, thermal desorption of a gas from a heterogeneous surface gives rise to very different desorption curves from those resulting for desorption from a homogeneous surface. Heterogeneity can be caused by the adsorbent surface or by lateral interaction between adsorbed particles. Fractional, zero, or very high-order desorption kinetics can result from a first-order desorption mechanism with an activation energy and a preexponential factor that depend on the kind of lattice site where the adatom desorption occurs, or from the above mentioned lateral interaction. The simplest method in dealing with heterogeneity is to assume an activation energy which is a function of coverage. A variety of empirical functions have been used ${ }^{1-3}$ and they have been useful in many cases. However, this method does not allow us to gain insight into the various mechanisms that make activation energy dependent on coverage.

Our purpose in this paper is to study the way in which substrate heterogeneity and lateral interaction between adatoms influence the desorption process. We start from a master equation, where transition probabilities are taken in the Arrhenius form. We consider two contributions to activation energy, namely, the energy due to the substrate and that due to lateral interaction between nearest-neighbor adatoms on the surface.

We have carried out an analysis of the desorption process for a variety of conditions. First we consider a linear chain where there are sites characterized with different activation energies due to the substrate and governed by a random distribution. The lateral interaction energy is a given constant and the Bragg-William approximation ${ }^{4}$ is used. Then we extend the formulation to treat lattices with any coordination number $c$. Later we study the case where the sites with the same activation energy are distributed periodically (periodical heterogeneous chain) and, subsequently, we consider a random patchwise model. Finally, we discuss our results and summarize our conclusions.

\section{RANDOM SITE DISTRIBUTION}

Firstly, we consider a linear chain with $N$ sites $(N \rightarrow \infty)$ where each site can be empty or occupied by one adatom. We associate a variable $s_{j}$ with each chain site, which takes the values 1 (filled site) or -1 (empty site). A state of the chain can be described by a set $\left\{s_{j}\right\}(j=1, \ldots, N)$ and we denote $P\left(s_{1}, \ldots, s_{N} ; t\right)$ the probability that the system is found in configuration $\left\{s_{1}, \ldots, s_{N}\right\}$ at time $t$. Two terms contribute to the activation energy, namely, a nearest-neighbor interaction energy $h_{0}$, given as constant, and an energy due to the substrate causing the heterogeneity. For simplicity we develop the model according to the case in which there are two types of sites, called 1 and 2 , which contribute to the activation energy with energies $h_{1}$ and $h_{2}$, respectively. Such a model is similar to a "heterogeneous" Ising model. ${ }^{5}$

We assume that only the desorption mechanism is operative, and readsorption onto the chain and migration of adatoms from site to site on the chain are inhibited. When a particle is desorbed from the $j$ th site, the value of $s_{j}$ changes from 1 to -1 . The time dependence of $P\left(s_{1}, \ldots, s_{N} ; t\right)$ can be described by the master equation 


$$
\begin{aligned}
\frac{d P\left(s_{1}, \ldots, s_{j}, \ldots, s_{N} ; t\right)}{d t}= & -\left[\sum_{j} W\left(s_{j-1}, s_{j}, s_{j+1}\right)\right] P\left(s_{1}, \ldots, s_{j}, \ldots, s_{N} ; t\right) \\
& +\sum_{j}\left[W\left(s_{j-1},-s_{j}, s_{j+1}\right) P\left(s_{1}, \ldots,-s_{j}, \ldots, s_{N} ; t\right)\right],
\end{aligned}
$$

where $W\left(s_{j-1}, s_{j}, s_{j+1}\right)$, which depends on $s_{j-1}, s_{j}$, and $s_{j+1}$ because of nearest-neighbor interaction, is the transition probability per unit time from state $s_{j}$ to state $-s_{j}$, while the other $s_{k}(k \neq j)$ variables remain temporarily fixed. These transition probabilities are taken in the Arrhenius form. There are three different probabilities, as shown in Fig. 1,

$$
\begin{aligned}
& W_{++}^{i}=A_{i} \exp \left[-\left(h_{i}+2 h_{0}\right) / k_{B} T\right], \\
& W_{+-}^{i}=W_{-+}^{i}=A_{i} \exp \left[-\left(h_{i}+h_{0}\right) / k_{B} T\right], \\
& W_{--}^{i}=A_{i} \exp \left(-h_{i} / k_{B} T\right),
\end{aligned}
$$

where superscript $i(i=1,2)$ indicates whether the central site is of type 1 or of type $2, A_{i}$ is the preexponential factor, $k_{B}$ is the Boltzmann constant, and $T$ is the temperature.

We define the following correlation functions:

$$
\begin{aligned}
& Q_{k}=\frac{1}{N}\left\langle\sum_{j}^{k} s_{j}\right\rangle, \\
& R_{k m}=\frac{1}{N}\left\langle\sum_{j}^{m} s_{j-1}^{k}\right\rangle, \\
& S_{k m}=\frac{1}{N}\left\langle\sum_{j}^{m} s_{j-1}^{k} s_{j}^{m}\right\rangle, \\
& T_{k m n}=\frac{1}{N}\left\langle\sum_{j}^{m} s_{j-1}^{k} s_{j+1}^{n}\right\rangle, \\
& U_{k m n}=\frac{1}{N}\left\langle\sum_{j}^{m} s_{j-1}^{k} s_{j}^{m} s_{j+1}^{n}\right\rangle,
\end{aligned}
$$

where

$$
\left\langle f\left(s_{k}\right)\right\rangle=\sum_{\left\{s_{j}\right\}} f\left(s_{k}\right) P\left(\left\{s_{j}\right\} ; t\right)
$$

and the sum $\sum_{j}^{i}(i=1,2)$ extends over all chain sites of type $i$. Also, when suitable for clarity, we have written $s_{j}^{i}(i=1,2)$ instead of $s_{j}$ to indicate that the $j$ th site is of type $i$.

From Eqs. (1) and (2) we obtain the kinetic equations

$$
\begin{aligned}
\frac{d Q_{i}}{d t}=-\frac{1}{4}[ & n_{i} a_{1}^{i}+a_{1}^{i} Q_{i}+2 a_{2}^{i}\left(R_{1 i}+R_{2 i}\right)+2 a_{2}^{i}\left(S_{1 i}+S_{2 i}\right) \\
& \left.+a_{3}^{i}\left(T_{1 i 1}+2 T_{1 i 2}+T_{2 i 2}\right)+a_{3}^{i}\left(U_{1 i 1}+2 U_{1 i 2}+U_{2 i 2}\right)\right], \quad i=1,2
\end{aligned}
$$

where $n_{i}$ indicates the fraction of sites of type $i$ in the chain $\left(n_{1}+n_{2}=1\right)$ and

$$
\begin{aligned}
& a_{1}^{i}=A_{i} e^{-h_{i} / k_{B} T}\left(e^{-h_{0} / k_{B} T}+1\right)^{2}, \\
& a_{2}^{i}=A_{i} e^{-h_{i} / k_{B} T}\left(e^{-2 h_{0} / k_{B} T}-1\right), \\
& a_{3}^{i}=A_{i} e^{-h_{i} / k_{B} T}\left(e^{-h_{0} / k_{B} T}-1\right)^{2} .
\end{aligned}
$$

The adsorption degree on sites of type $i$ is

$$
\Theta_{i}=\frac{n_{i}+Q_{i}}{2}
$$

and the total adsorption degree is

$$
\Theta=\sum_{i} \Theta_{i}
$$

Because Eqs. (5) involve correlation functions comprising more than one chain site, we need to ap- 


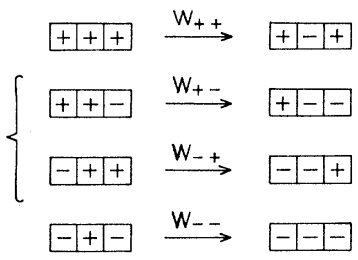

FIG. 1. Schematic diagram of the removal probabilities.

ply some method of approximation to solve these equations. The simplest method is the BraggWilliam approximation. More refined approximations can be carried out, ${ }^{6-9}$ but then calculations become more cumbersome. Here we shall apply the Bragg-William approximation.

If $i, j, k$ denote the plus or minus sign (plus and minus signs indicate filled and empty sites, respectively) and $m, n, p$ take the values 1 and 2 ( 1 indicates site of type $1 ; 2$ indicates site of type 2 ), let $N_{i j}^{m n}$ be the fraction of nearest site pairs, the first being a site $m$ in a state $i$ and the second a site $n$ in a state $j$ (for example, $\mathrm{N}_{-+}^{12}$ denotes the fraction of site pairs, the first being an empty site of type 1 and the second a filled site of type 2), and let $N_{i j k}^{m n p}$ be the fraction of triplets of sites of types $m, n, p$ in states $i, j, k$, respectively. We assume that

$$
\begin{aligned}
& N_{i j}^{m n}=N_{i}^{m} N_{j}^{n}, \\
& N_{i j k}^{m n p}=N_{i}^{m} N_{j}^{n} N_{k}^{p},
\end{aligned}
$$

$N_{i}^{m}$ being the fraction of sites of type $m$ in state $i$.

With this assumption, and by means of a straightforward but tedious calculation, Eqs. (5) become

$$
\begin{aligned}
\frac{d \Theta_{i}}{d t}= & -A_{i} e^{-h_{i} / k_{B} T} \Theta_{i} \\
& \times\left[1+\left(e^{-h_{0} / k_{B} T}-1\right) \Theta\right]^{2}, \quad i=1,2 .
\end{aligned}
$$

For the total adsorption degree the result is

$$
\begin{aligned}
\frac{d \Theta}{d t}=- & \left(A_{1} e^{-h_{1} / k_{B} T} \Theta_{1}+A_{2} e^{-h_{2} / k_{B} T} \Theta_{2}\right) \\
& \times\left[1+\left(e^{-h_{0} / k_{B} T}-1\right) \Theta\right]^{2} .
\end{aligned}
$$

Now it is very easy to extend the above equations for the case of $n$ different types of sites and for a continuous distribution of types of sites (see the Appendix).

When the chain is homogeneous, we find

$$
\frac{d \Theta}{d t}=-A e^{-h / k_{B} T}\left[1+\left(e^{-h_{0} / k_{B} T}-1\right) \Theta\right]^{2} .
$$

From Eqs. (11) - (13) it is apparent that the effect of nearest-neighbor interaction is involved only in the factor

$$
\left[1+\left(e^{-h_{0} / k_{B} T}-1\right) \Theta\right]^{2} .
$$

If $h_{0}=0$, this factor equals 1 and Eq. (13) becomes the standard Arrhenius equation.

If

$$
\exp \left(-h_{0} / k_{B} T\right) \Theta \gg 1-\theta
$$

(strongly repulsive interaction), we can write Eq. (13) as

$$
\frac{d \Theta}{d t}=-A \exp \left[-\left(h+2 h_{0}\right) / k_{B} T\right] \Theta^{3}
$$

(third-order kinetics), which is an equation similar to the Arrhenius equation with

$$
E_{\text {act }}=h+2 h_{0}-2 k_{B} T \ln \Theta .
$$

If $\exp \left(-h_{0} / k_{B} T\right)<1$ (strongly attractive interaction), Eq. (13) becomes

$$
\frac{d \Theta}{d t}=-A \exp \left(-h / k_{B} T\right) \Theta(1-\Theta)^{2} .
$$

Note that while in Eq. (14) $d \theta / d t$ is proportional to $\left(N_{+} / N\right)^{3}$ (i.e., to $N_{+++} / N$ in the Bragg-William approximation), in Eq. (15) it is proportional to $\left(N_{+} / N\right)\left(N_{-} / N\right)^{2}$ (i.e., to $N_{-+-} / N$ in the BraggWilliam approximation).

Turning now to the general problem, let us consider the heterogeneous case. By means of a straightforward calculation we obtain

$$
\Theta_{2}=n_{2}\left(\Theta_{1} / n_{1}\right)^{r \exp \left(-g / k_{B} T\right)}
$$

where $r=A_{2} / A_{1}$ and $g=h_{2}-h_{1}$. Then the adsorption degree must verify the following equations:

$$
\begin{aligned}
& \Theta=\Theta_{1}+n_{2}\left(\Theta_{1} / n_{1}\right)^{r \exp \left(-g / k_{B} T\right)}, \\
& \frac{d \Theta}{d t}=-A_{1} e^{-h_{1} / k_{B} T}\left[\Theta_{1}+r e^{-g / k_{B} T} n_{2}\left(\Theta_{1} / n_{1}\right)^{r \exp \left(-g / k_{B} T\right)}\right]\left[1+\left(e^{-h_{0} / k_{B} T}-1\right) \Theta\right]^{2}
\end{aligned}
$$


The time evolution of $\Theta$ can be found from Eqs. (17) and (18), at least formally, by elimination of $\Theta_{1}$. In general, these equations require a numerical solution.

If we consider now that adatoms are desorbed from a lattice with coordination number $c$ and we apply the Bragg-William approximation, we obtain

$$
\begin{aligned}
\frac{d \Theta_{i}}{d t}= & -A_{i} e^{-h_{i} / k_{B} T} \Theta_{i} \\
& \times\left[1+\left(e^{-h_{0} / k_{B} T}-1\right) \Theta\right]^{c}, \quad i=1,2 .
\end{aligned}
$$

Equations (19) and (11) are similar, except for the exponent of the factor due to interaction between nearest neighbors.

\section{PERIODICAL AND PATCHWISE HETEROGENEOUS SURFACES}

The above analysis is valid for the case where the distribution of different types of sites is purely random. We consider now the case where this distribution is not purely random, but where we have additional information about it. The simplest case is that of a periodical heterogeneous lattice, i.e., there are different types of sites but they are distributed in a periodical form.

Let two types of sites, 1 and 2, lie in a linear chain. We assume that the densities of each type of site $n_{1}$ and $n_{2}$, those of duplets $n_{i j}(i, j=1,2)$, and those of triplets $n_{i j k}(i, j, k=1,2)$ are known. In Fig. 2, two perodical heterogeneous linear chains are shown. For case (a) $n_{1}=n_{2}=\frac{1}{2}, n_{11}=n_{22}=0$, $n_{12}=n_{21}=\frac{1}{2}, \quad n_{121}=n_{212}=\frac{1}{2}, \quad n_{111}=n_{112}=n_{211}$ $=n_{221}=n_{122}=n_{222}=0$. For case (b) $n_{1}=\frac{2}{3}$, $n_{2}=\frac{1}{3}, n_{22}=0, n_{11}=n_{12}=n_{21}=\frac{1}{3}, n_{112}=n_{121}$ $=n_{211}=\frac{1}{3}, n_{111}=n_{212}=n_{221}=n_{122}=n_{222}=0$. When all sites 1 are clustered on one part and all sites 2 on another, $n_{11}=n_{1}, n_{22}=n_{2}, n_{12}=n_{21}=0$. For the case of random distribution previously considered, $\quad n_{11}=n_{1}^{2}, \quad n_{22}=n_{2}^{2}, \quad n_{12}=n_{21}=n_{1} n_{2}$, $n_{111}=n_{1}^{3}$, and so on.

Approximation given by Eqs. (9) and (10) may be

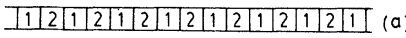

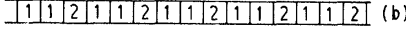

FIG. 2. Periodical heterogeneous linear chains: (a) alternating sites; (b) alternating pairs 1-1 and sites 2 . expressed as

$$
\begin{aligned}
& N_{i j}^{l m}=n_{l m} q_{i}^{l} q_{j}^{m}, \\
& N_{i j k}^{l m n}=n_{l m n} q_{i}^{l} q_{j}^{m} q_{k}^{n},
\end{aligned}
$$

where $q_{k}^{n}$ denotes the relative fraction of sites of type $n \quad(n=1,2)$ which are in the state $k$ $(k \rightarrow+,-)$. Then Eqs. (5) and (6), jointly with Eqs. (20) and (21), lead to

$$
\begin{aligned}
& \frac{d \Theta_{i}}{d t}=-A_{i} e^{-h_{i} / k_{B} T} \Theta_{i} \\
& \times\left(1+\frac{2 z}{n_{i}} \sum_{j} n_{i j} \frac{\Theta_{j}}{n_{j}}+\frac{z^{2}}{n_{i}} \sum_{j, k} n_{j i k} \frac{\Theta_{j}}{n_{j}} \frac{\Theta_{k}}{n_{k}}\right), \\
& i, j, k=1,2
\end{aligned}
$$

where $z=\exp \left(-h_{0} / k_{B} T\right)-1$.

Finally, we treat the case where there are different types of sites with a patchwise distribution. For the sake of simplicity we consider again two types of sites, 1 and 2, with densities $n_{1}$ and $n_{2}$, respectively, on a linear chain with $N$ sites. Patches of type 1 and of type 2 must be alternatively placed on this chain, $d$ denoting the number of patches of type 1 (or of type 2). Let $F_{1}(m)$ and $F_{2}(m)$ be the respective distribution functions of the patch sizes, i.e., $F_{i}(m)$ is the probability of finding a patch formed by $m$ consecutive sites of type $i$. Functions $F_{i}(m)$ verify

and

$$
\sum_{m} F_{i}(m)=1
$$

$$
\frac{n_{1}}{M_{1}}=\frac{n_{2}}{M_{2}}=\frac{d}{N}
$$

where

$$
M_{1}=\sum_{m} F_{1}(m) m, \quad M_{2}=\sum_{m} F_{2}(m) m,
$$

$M_{i}$ being the average size of type $i$ patches in the chain.

Densities of site groups involved in Eqs. (22), $n_{i}$, $n_{i j}, n_{i j k}(i, j, k=1,2)$ can be written as

$$
\begin{aligned}
n_{1} & =\frac{M_{1}}{M_{1}+M_{2}}=\frac{d}{N} M_{1}, \\
n_{2} & =\frac{M_{2}}{M_{1}+M_{2}}=\frac{d}{N} M_{2}, \\
n_{11} & =\frac{d}{N} \sum_{m}(m-1) F_{1}(m) \\
& =n_{1}-\frac{d}{N} \sum_{m} F_{1}(m)=n_{1}-\frac{n_{1}}{M_{1}},
\end{aligned}
$$


and so on. Substituting Eqs. (26) into Eqs. (22), one gets the kinetic equations governing the desorption process.

Extension of the above formulation to twodimensional lattices is difficult because in order to characterize patches one must specify not only their size, but also their form, and then the problem becomes impracticable because the number of possibilities is enormous. However, if one introduces restrictions on the patch forms or knows the distribution of clusters involving a site and its nearest neighbors, for instance, the cluster

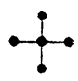

for square lattices, it is possible to treat the problem in the same way as for the linear chain, although calculations become cumbersome.

\section{RESULTS AND DISCUSSION}

To analyze the way in which lateral interaction and substrate heterogeneity influence the rate desorption, we have carried out numerical calculations for several specific cases. We have assumed a temperature-programmed desorption and a linear relationship between temperature $T$ and time $t$, i.e., $T=T_{0}+u t$, where $u$ is a constant. We have taken $A / u=5 \times 10^{6} \mathrm{~K}^{-1}$.

Firstly, we consider a homogeneous chain [Eq. (13)] with attractive, zero, and repulsive lateral interaction energy. The results are shown in Fig. 3. The effect of lateral interaction on the curve $d \Theta / d T$ vs $T$ is clear. Repulsive interaction energy results in the desorption starting at a lower tem-

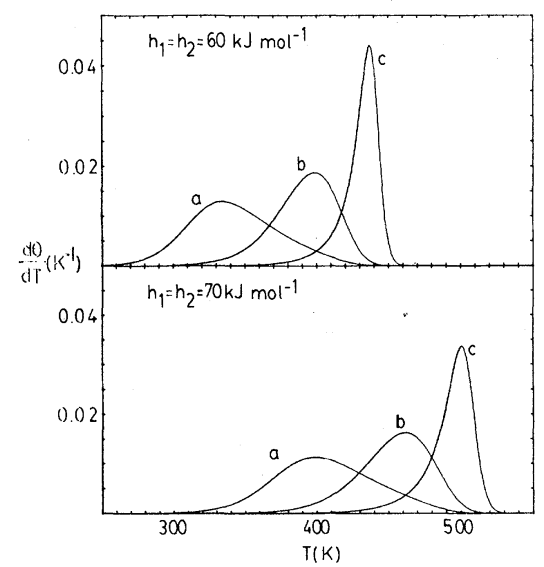

FIG. 3. Desorption rate vs temperature for a homogeneous chain: (a) $h_{0}=-5 \mathrm{~kJ} \mathrm{~mol}^{-1}$; (b) $h_{0}=0$; (c) $h_{0}=5$ $\mathrm{kJ} \mathrm{mol}^{-1}$. perature while occurring more smoothly (i.e., the curve $d \Theta / d T$ vs $T$ exhibits a less sharp maximum) than when interaction does not exist. On the contrary, attractive interaction energy results in the desorption starting at a higher temperature while occurring in a temperature range more narrow than in the above cases, and the curve $d \Theta / d T$ vs $T$ exhibits a very sharp maximum. Similar results are again shown in Fig. 4, where we present the curve $d \Theta / d T$ vs $T$ for a heterogeneous chain, where two types of sites 1 and 2 are considered, with $n_{1}=n_{2}=0.5$, and distributed randomly $\left(n_{i j}=n_{i} n_{j}\right.$, $n_{i j k}=n_{i} n_{j} n_{k}$ ). Heterogeneity yields two maxima in the curve $d \Theta / d T$ vs $T$, the maximum at low temperature being the sharpest.

Results more interesting than the above ones are obtained when we analyze the influence that the heterogeneity distribution on the chain exerts on the curve $d \Theta / d T$ vs $T$. In Figs. 5 and 6 we show results obtained for a chain with two types of sites, with $n_{1}=n_{2}=0.5$, for four different site distributions: (a) Regular or periodical chain, like the chain shown in Fig. 2 (a); (b) random site distribution, with $n_{i j}=n_{i} n_{j}$ and $n_{i j k}=n_{i} n_{j} n_{k}$; (c) patchwise site distribution, where we assume that the patch size is governed by a Poisson distribution with average size fixed (for the calculations this average size has been taken as equal to ten sites); (d) distribution in two domains, each domain comprising the sites of type 1 or of type 2 , respectively. In Fig. 5 results for attractive lateral interaction are shown. The curve $d \Theta / d T$ vs $T$ exhibits only one maximum for case (a) and two maxima for the others. The difference between the temperatures corresponding to maxima of $d \Theta / d T$ and the sharpness of these maxima increases as the number of pairs $1-2, n_{12}$, decreases, case (d) being the extreme case. In Fig. 6 results for repulsive lateral interaction are plotted. Now the

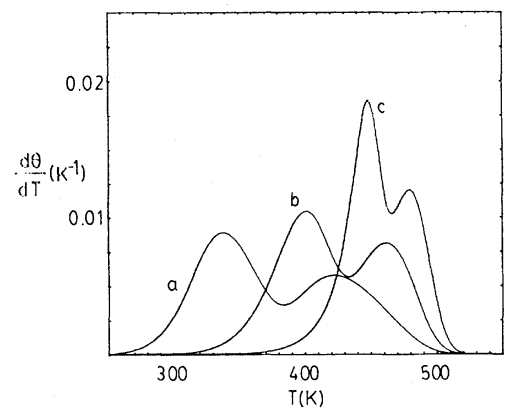

FIG. 4. Desorption rate vs temperature for a random heterogeneous linear chain, with $n_{1}=n_{2}=0.5, h_{1}=60$ $\mathrm{kJ} \mathrm{mol}^{-1}, h_{2}=70 \mathrm{~kJ} \mathrm{~mol}^{-1}$, and: (a) $h_{0}=-5 \mathrm{~kJ} \mathrm{~mol}^{-1}$; (b) $h_{0}=0$; (c) $h_{0}=5 \mathrm{~kJ} \mathrm{~mol}^{-1}$. 


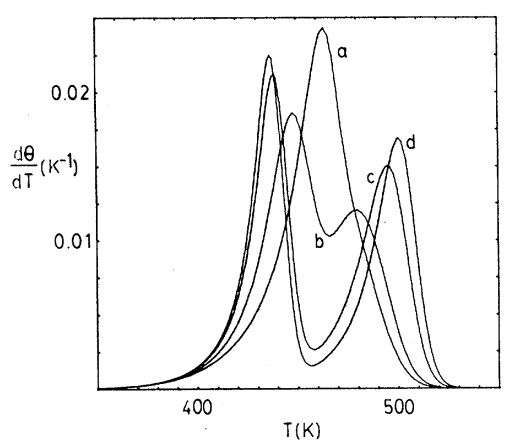

FIG. 5. Desorption rate vs temperature for a heterogeneous linear chain with $n_{1}=n_{2}=0.5$ and $h_{1}=60$ $\mathrm{kJ} \mathrm{mol}^{-1}, h_{2}=70 \mathrm{~kJ} \mathrm{~mol}^{-1}, h_{0}=5 \mathrm{~kJ} \mathrm{~mol}^{-1}$ : (a) periodical chain; (b) random chain; (c) patches with average size equal to ten sites; (d) distribution in two domains, each domain comprising the sites of type 1 or of type 2, respectively.

results are inverted with regard to the above ones. In fact, the maxima are the sharpest and the most distant for case (a) and succesively sharp and distant for case (b). For cases (c) and (d) the difference between the values of the maxima is small and the central part of the curve $d \Theta / d T$ is very smooth and nearly flat. For case (d) the maximum at high temperature is slightly higher than the maximum at low temperature. By comparison of Fig. 5 and 6, it can also be seen that for repulsive lateral interaction desorption occurs within a larger temperature range with maxima less sharp than for attractive lateral interactions, as we have indicated above.

In conclusion, we can remark that when substrate heterogeneity and lateral interaction between ada-

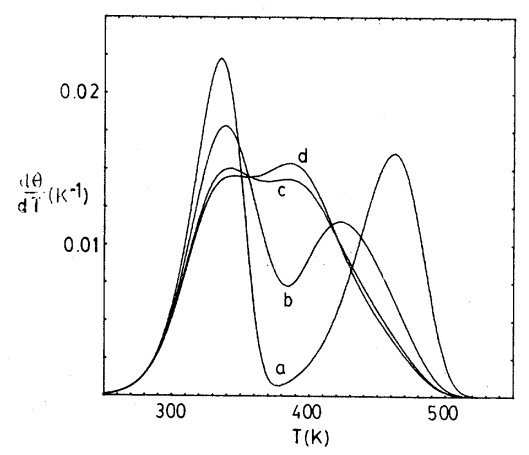

FIG. 6. Desorption rate vs temperature for a heterogeneous linear chain with $n_{1}=n_{2}=0.5$ and $h_{1}=60$ $\mathrm{kJ} \mathrm{mol}^{-1}, \quad h_{2}=70 \quad \mathrm{~kJ} \mathrm{~mol}^{-1}, \quad h_{0}=-5 \quad \mathrm{~kJ} \mathrm{~mol}^{-1}$ : (a) Periodical chain; (b) random chain; (c) patches with average size equal to ten sites; (d) distribution in two domains, each domain comprising the sites of type 1 or of type 2 , respectively. toms coexist, the desorption curve $d \Theta / d T$ vs $T$ depends strongly on the type and fraction of adsorbent sites and also on the distribution of these sites on the chain (periodically, patches with different sizes, etc.). This fact makes it clear that lateral interaction can soften or strengthen substrate heterogeneities and accordingly, in order to clarify adsorption-desorption mechanisms, we recognize the need for characterizing the substrate heterogeneity distribution in the most accurate way possible, independently of lateral interaction between adatoms.

\section{APPENDIX}

We can extend the equations in Sec. II to the case of $n$ different types of sites, $n_{i}$ being the fraction of sites of type $i$ on the chain. Equations (11) and (12) become

$$
\begin{aligned}
& \frac{d \Theta_{i}}{d t}=-A_{i} e^{-h_{i} / k_{B} T} \Theta_{i} \\
& \times\left[1+\left(e^{-h_{0} / k_{B} T}-1\right) \Theta\right]^{2}, \\
& i=1, \ldots, n \\
& \frac{d \Theta}{d t}=-\left(\sum_{i=1}^{n} A_{i} e^{-h_{i} / k_{B} T} \Theta_{i}\right) \\
& \times\left[1+\left(e^{-h_{0} / k_{B} T}-1\right) \Theta\right]^{2},
\end{aligned}
$$

and then

$$
\begin{gathered}
\Theta_{i}=n_{i}\left(\Theta_{1} / n_{1}\right)^{r_{i} \exp \left(-g_{i} / k_{B} T\right)}, \\
\Theta=\sum_{i} n_{i}\left(\Theta_{i} / n_{i}\right)^{r_{i} \exp \left(-g_{i} / k_{B} T\right)}, \\
\frac{d \Theta}{d t}=-\left(\sum_{i} A_{i} n_{i} e^{-h_{i} / k_{B} T}\right. \\
\left.\times\left(\Theta_{1} / n_{1}\right)^{r_{i} \exp \left(-g_{i} / k_{B} T\right)}\right) \\
\times\left[1+\left(e^{-h_{0} / k_{B} T}-1\right) \Theta\right]^{2},
\end{gathered}
$$

where $r_{i}=A_{i} / A_{1}$ and $g_{i}=h_{i}-h_{1}$.

If instead of a discrete distribution of types of sites, we consider a continuous distribution so that $f(h) d h$ is the fraction of sites with energy comprised between $h_{1}+h$ and $h_{1}+h+d h$, the sums on the right-hand side of Eqs. (A4) and (A5) must be substituted by the corresponding integrals 


$$
\begin{gathered}
\Theta=\int f(h)\left(\Theta_{1} / n_{1}\right)^{r(h) \exp \left(-h / k_{B} T\right)} d h, \\
\frac{d \Theta}{d t}=-\iint A(h) e^{-\left(h_{1}+h\right) / k_{B} T} \\
\left.\times\left(\Theta_{1} / n_{1}\right)^{r(h) \exp \left(-h / k_{B} T\right)} f(h) d h\right] \\
\times\left[1+\left(e^{-h_{0} / k_{B} T}-1\right) \Theta\right]^{2} .
\end{gathered}
$$

To solve Eqs. (A6) and (A7) one needs to know $A(h)$ and $f(h)$. So in the case described by Eqs. (A4) and (A5) as in the case described by Eqs. (A6) and (A7), it is not possible to find an exact solution. Indeed, even for the most simplified cases, the resulting equations require a numerical solution. To illustrate the difficulties appearing in this problem, we consider the following continuous cases: $A(h)=A=$ const, i.e., $r=1$, and

$$
f(h)=\left\{\begin{array}{l}
0, \quad h<0 \\
M^{-1}, 0 \leq h \leq M \\
0, \quad h>M
\end{array}\right.
$$

In this case, we obtain

$$
\Theta=1-\frac{k_{B} T}{M} \sum_{n=1}^{\infty} \frac{\left(e^{-n M / k_{B} T}-1\right) \ln ^{n}\left(\Theta_{1} / n_{1}\right)}{n n !}
$$

$$
\begin{aligned}
\frac{d \Theta}{d t}= & \frac{A e^{-h_{1} / k_{B} T}}{M} \frac{k_{B} T}{\ln \left(\Theta_{1} / n_{1}\right)} \\
& \times\left[\left[\frac{\Theta_{1}}{n_{1}}\right]^{\exp \left(-M / k_{B} T\right)}-\frac{\Theta_{1}}{n_{1}}\right] \\
& \times\left[1+\left(e^{-h_{0} / k_{B} T}-1\right) \Theta\right]^{2} .
\end{aligned}
$$

Because $\Theta_{1} / n_{1}$ cannot be eliminated between Eq. (A8) and Eq. (A9), we must start from

$$
\frac{d\left(\Theta_{1} / n_{1}\right)}{d t}=-A e^{-h_{1} / k_{B} T} \frac{\Theta_{1}}{n_{1}}\left\{1+\left(e^{-h_{0} / k_{B} T}-1\right)\left[1-\frac{k_{B} T}{M} \sum_{n=1}^{\infty}\left[\frac{e^{-n M / k_{B} T} \ln ^{n}\left(\Theta_{1} / n_{1}\right)}{n n !}-\frac{\ln ^{n}\left(\Theta_{1} / n_{1}\right)}{n n !}\right]\right]\right.
$$

Then we obtain the value of $\Theta_{1} / n_{1}$ at time $t$, truncating the series involved in Eq. (A10) in an order depending on the required accuracy. After we have found $\Theta_{1} / n_{1}$, we can substitute it into Eq. (A8) and get the value of $\Theta$ at time $t$.

${ }^{1}$ See, for example, J. G. Dash, Films on Solid Surfaces (Academic, New York, 1975), Chap. 9 and references therein; A. W. Adamson, Physical Chemistry of Surfaces (Wiley-Interscience, New York, 1976), Chap. $\mathrm{XIV}$ and references therein.

${ }^{2}$ B. Kindl, R. A. Pachovsky, B. A. Spencer, and B. W. Wojciechowsky, J. Chem. Soc. Faraday Trans. 1 69, 1162 (1973).

${ }^{3}$ Y. Tokoro, T. Uchijima, and Y. Yoneda, J. Catal. 뜨, 110 (1979).

${ }^{4}$ See, for example, K. Huang, Statistical Mechanics (Wi- ley, New York, 1963), Chap. 16.

5I. Morgensten, K. Binder, and A. Baumgartner, J. Chem. Phys. 69, 253 (1978) and references therein; I. Morgensten, K. Binder, and R. M. Hornreich, Phys. Rev. B 23, 287 (1981) and references therein.

${ }^{6} \mathrm{G}$. Schwarz, Ber. Bunsenges. Phys. Chem. 75, 40 (1971); J. Theor. Biol. 느, 569 (1972).

${ }^{7}$ H. W. Huang, Phys. Rev. A $\underline{8}, 2553$ (1973).

${ }^{8}$ Y. Saito and R. Kubo, J. Stat. Phys. 15, 233 (1976).

9J. J. Luque and A. Córdoba, J. Chem. Phys. 76, 6393 (1982). 